\title{
Instantaneous Drug Delivery of Magnetic/Thermally Sensitive Nanospheres by a High-Frequency Magnetic Field
}

\author{
Ting-Yu Liu, Shang-Hsiu Hu, Kun-Ho Liu, Ren-Shiuan Shaiu, Dean-Mo Liu, * and \\ San-Yuan Chen*
} Department of Materials Sciences and Engineering, National Chiao Tung University,
Hsinchu, Taiwan, 300, Republic of China

Received May 10, 2008. Revised Manuscript Received July 2, 2008

\begin{abstract}
Novel dual-functional nanospheres composed of magnetic iron oxide nanoparticles embedded in a thermo-sensitive Pluronic F127 (F127) matrix were successfully synthesized by an in situ coprecipitation process. The nanospheres were characterized by X-ray diffraction, transmission electron microscopy, X-ray photoelectron spectroscopy, and Raman spectroscopy. Experimental observations indicated that the F127 was subjected to a rapid structural change when the magnetic phase caused rapid heating after a short exposure to a high-frequency magnetic field. During the field duration, considerable volume shrinkage of the nanospheres (2.3-fold diameter reduction) was detected. This has been translated to an instantaneous release of a drug, Doxorubicin (DOX), when the DOX was encapsulated within the nanospheres. Such a rapidly responsive release of the DOX from the nanospheres was due to an intimate contact between the nanomagnet and F127, where an effective thermal and mechanical transfer between core and shell phases efficiently took place in the presence of the magnetic field.
\end{abstract}

\section{Introduction}

Drug carriers that are responsive to external stimuli, such as temperature, $\mathrm{pH}$, light, mechanical signal, electric field, and magnetic fields, have received great attention in recent years. ${ }^{1-9}$ A wide variety of materials, such as carbon nanotubes, dendrimers, biodegradable nanoparticles (poly(lactide-co-glycolic acid) (PLGA) or poly(lactic acid) (PLA)), lipid vesicles (micelles), and gold or magnetic nanoparticles have been employed as matrix materials to deliver drugs. Among them, magnetic nanoparticles (MNPs) provide more interesting opportunities since they can be effectively activated in a controllable manner through a noncontact stimulus, ${ }^{10}$ as compared with other materials. Although gold nanoparticles also displayed a similar inductive heating ability by infrared light stimulus, the manipulation and heating ability of MNPs were superior to those of the gold nanoparticles. The ability of magnets to convert magnetic energy into heat by the hysteresis effect has been known for a long period of time; ${ }^{11}$ for instance, the ability has been used as antenna material for inductive heating in antitumor therapy, but the efficiency has not been as

* Corresponding author. Address: Department of Materials Sciences and Engineering, National Chiao Tung University 1001 Ta Hsueh Road, Hsinchu, Taiwan 300, ROC. Telephone: +886-3-5712121ext. 31818. Fax: +886-35725490. E-mail:sanyuanchen@mail.nctu.edu.tw (S.-Y.C.); deanmo_liu@ yahoo.ca (D.-M.L.).

(1) Kim, M. R.; Park, T. G. J. Controlled Release 2002, 104, 69-77.

(2) Zhang, X. Z.; Wu, D. Q.; Chu, C. C. Biomaterials 2004, 25, 3793-3805.

(3) Chiu, G. N. C.; Abraham, S. A.; Ickenstein, L. M.; Ng, R.; Karlsson, G.; Edwards, K.; Wasan, E. K.; Bally, M. B. J. Controlled Release 2005, 104, 271288.

(4) Lu, Z.; Prouty, M. D.; Guo, Z.; Golub, V. O.; Kumar, C. S. S. R.; Lvov, Y. M. Langmuir 2005, 21, 2042-2050.

(5) Qiu, Y.; Park, K. Adv. Drug Delivery Rev. 2001, 53, 321-339.

(6) Etrych, T.; Jelínková, M.; Øíhová, B.; Ulbrich, K. J. Controlled Release 2001, 73, 89-102.

(7) Chen, S. C.; Wu, Y. C.; Mi, F. L.; Lin, Y. H.; Yu, L. C.; Sung, H. W. Controlled Release 2004, 96, 285-300.

(8) Murdan, S. J. Controlled Release 2003, 92, 1-17.

(9) Mamada, A.; Tanaka, T.; Kungwatchakun, D.; Irie, M. Macromolecules 1990, 23, 1517-1519.

(10) Kohler, N.; Sun, C.; Fichtenholtz, A.; Gunn, J.; Fang, C.; Zhang, M. Small 2006, 2, 785-792.

(11) Kim, D. H.; Lee, S. H.; Kim, K. N.; Kim, K. M.; Shim, I. B.; Lee, Y. K. J. Magn. Magn. Mater. 2005, 293, 320-327. good as desired without combining inductive heating with drug delivery. In order to enhance the tumor-inhibition ability, the combination of the heat transfer and drug release in nanoparticles will be more interesting. In our previous work, we developed magnetic hydrogels (ferrogels) ${ }^{12-14}$ and magnetically sensitive nanospheres for controlled drug release by a high-frequency magnetic field (HFMF) ${ }^{15,16}$ However, the silica seems to be a thermally insensitive material, and it was believed that the nanostructure did not change with temperature increases. In order to accelerate the rate of drug release, a thermosensitive polymer was used in this system because it exhibited a huge volume change when the temperature was increased. ${ }^{17}$

A number of polymeric materials are known to exhibit a discontinuous change of properties when subjected to a temperature change. ${ }^{17-23}$ Some of these systems, e.g., thermoreversible gels, have been based on the existence of a lower critical solution temperature (LCST), or critical micellization temperature (CMT), and have usually been applied as intelligent drug carriers. ${ }^{17-23} \operatorname{Poly}(N$-isopropylacrylamide) (PNIPAAm), poly(ethylene glycol) - poly(lactic acid) - poly(ethylene glycol) triblocks (PEG-PLA-PEG), and poly(ethylene oxide)-poly(propylene oxide)-poly(ethylene oxide) (PEO-PPO-PEO)

(12) Liu, T. Y.; Hu, S. H.; Liu, T. Y.; Liu, D. M.; Chen, S. Y. Langmuir 2006, $22,5974-5978$.

(13) Hu, S. H.; Liu, T. Y.; Liu, D. M.; Chen, S. Y. J. Controlled Release 2007, 121, 181-189.

(14) Liu, T. Y.; Hu, S. H.; Liu, K. H.; Liu, D. M.; Chen, S. Y. J. Controlled Release 2008, 126, 228-236.

(15) Hu, S. H.; Liu, T. Y.; Liu, D. M.; Chen, S. Y. Macromolecules 2007, 40, 6786-6788.

(16) Hu, S. H.; Liu, T. Y.; Huang, H. Y.; Liu, D. M.; Chen, S. Y. Langmuir 2008, 24, 239-244.

(17) Choi, S. H.; Lee, J. H.; Choi, S. M.; Park, T. G. Langmuir 2006, 22 , $1758-1762$.

(18) Schmidt, A. M. Colloid Polvm. Sci. 2007, 285, 953-966.

(19) Coughlan, D. C.; Quilty, F. P.; Corrigan, O. I. J. Controlled Release 2004, 98, 97-114.

(20) Bhattacharya, S.; Eckert, F.; Boyko, V.; Pich, A. Small 2007, 3, 650-657. (21) Li, Y. Y.; Zhang, X. Z.; Kim, G. C.; Cheng, H.; Cheng, S. X.; Zhuo, R. X. Small 2006, 2, 917-923.

(22) Bae, K. H.; Lee, Y.; Park, T. G. Biomacromolecules 2007, 8, 650-656.

(23) Desai, P. R.; Jain, N. J.; Sharma, R. K.; Bahadur, P. Colloid Surf., A 2001, $178,57-69$. 
triblocks have been among the most important reverse thermal gelation-displaying polymers. These triblocks, especially Poloxamer 407, also known by the trade name Pluronic F127 $\left(\mathrm{PEO}_{100}-\mathrm{PPO}_{65}-\mathrm{PEO}_{100}\right)$, have been investigated for wound covering and controlled drug delivery. Furthermore, Pluronic series copolymer has excellent biocompatibility and is one of the very few synthetic polymers approved by the US Food and Drug Administration (FDA) for use as a food additive and pharmaceutical ingredient. $^{22,23}$ Pluronic series copolymer is also a potential candidate for biomedical applications.

In the present work, a dual-functional nanoparticle was developed by integrating a nanomagnetic core with a shell layer of thermosensitive hydrogel to encapsulate an anticancer agent. Inductive heat was generated in the presence of HFMF. When heated, the thermosensitive polymer collapsed around the iron oxide nanoparticles, inducing an accelerative drug release. In recent years, some researchers ${ }^{24-27}$ have reported that dualfunctional drug carriers included magnetic/thermal sensitivity, but few reports emphasized controlled drug release using HFMF. In addition, raising the temperature of the drug carriers from the surrounding heat source has been insufficient; a better alternative would be to supply the heat source by the drug carrier, e.g., the inductive heating effect induced by HFMF to a magnet core. This type of drug carrier can have great potential for disease treatment.

Moreover, the use of a high-frequency magnetic stimulus can also achieve an "instantaneous" burst release of drug by rapid heating to induce an instant shrinkage of the F127 hydrogel. Choi et al. ${ }^{17}$ reported that Pluronic/Heparin nanocapsules exhibit a 1000-fold thermally reversible volume transition when the temperature changes from 25 to $37{ }^{\circ} \mathrm{C}$. The violent volumeshrinkage would induce rapid drug release. Therefore, the combination of magnetic and thermal properties would be a very interesting alternative for tumor therapy. Here, we developed a novel Pluronic/iron oxide MNP formulated by an in situ coprecipitation process and evaluated it as a drug carrier. The proposed synthesis process of F127-MNPs is depicted in Figure 1a and is characterized by transmission electron microscopy (TEM), X-ray diffraction (XRD), Raman spectroscopy, X-ray photoelectron spectroscopy (XPS), spectrofluorophotometry (PL), and dynamic light scattering (DLS). Thereby, we demonstrated an approach to form water-dispersible and magnetic/thermoresponsive nanoparticles formulated with F127 and iron oxide nanoparticles, which can be applied to biomedical devices such as drug carriers.

\section{Experiment}

2.1. Procedures. Nanospheres of Pluronic F127 (F127) and MNPs (F127-MNPs) were synthesized using an in situ coprecipitation of $\mathrm{Fe}(\mathrm{II})$ and $\mathrm{Fe}(\mathrm{III})$ salts in the presence of Pluronic F127 (F127, Sigma). In this process, the optimum ratio of iron oxide precursor and F127 hydrogel was described: $0.05 \mathrm{~g}$ of F127, $5 \mathrm{mmol}$ of $\mathrm{FeCl}_{3} \cdot 6 \mathrm{H}_{2} \mathrm{O}$ (Riedel-deHaën), and 2.5 mmole of $\mathrm{FeCl}_{2} \cdot 4 \mathrm{H}_{2} \mathrm{O}$ (Fluka) were dissolved in $50 \mathrm{~mL}$ of water while being vigorously stirred at $60^{\circ} \mathrm{C}$. The dissolved solution was sonicated by a horn-type ultrasonic homogenizer (VC-500 W, Cole-Parmer Instruments, USA) operating at a constant frequency of $20 \mathrm{kHz}$ for $10 \mathrm{~min}$. Then, Pluronic F127/ iron oxide salt nanospheres were prepared, as shown in Figure 1-(a1) (where pure F127 nanospheres (appropriate size: $10-30 \mathrm{~nm}$ ) were

(24) Zhang, J. L.; Srivastava, R. S.; Misra, R. D. K. Langmuir 2007, 23, 6342-6351.

(25) Chen, S.; Li, Y.; Guo, C.; Wang, J.; Ma, J.; Liang, X.; Yang, L. R.; Liu, H. Z. Langmuir 2007, 23, 12669-12676.

(26) Deng, Y.; Wang, C.; Shen, X.; Yang, W.; Jin, L.; Gao, H.; Fu, S. Chem.-Eur. J. 2005, 11, 6006-6013.

(27) Detlef, M. S.; Thomas, S. R. J. Magn. Magn. Mater. 2006, 302, 267-271.

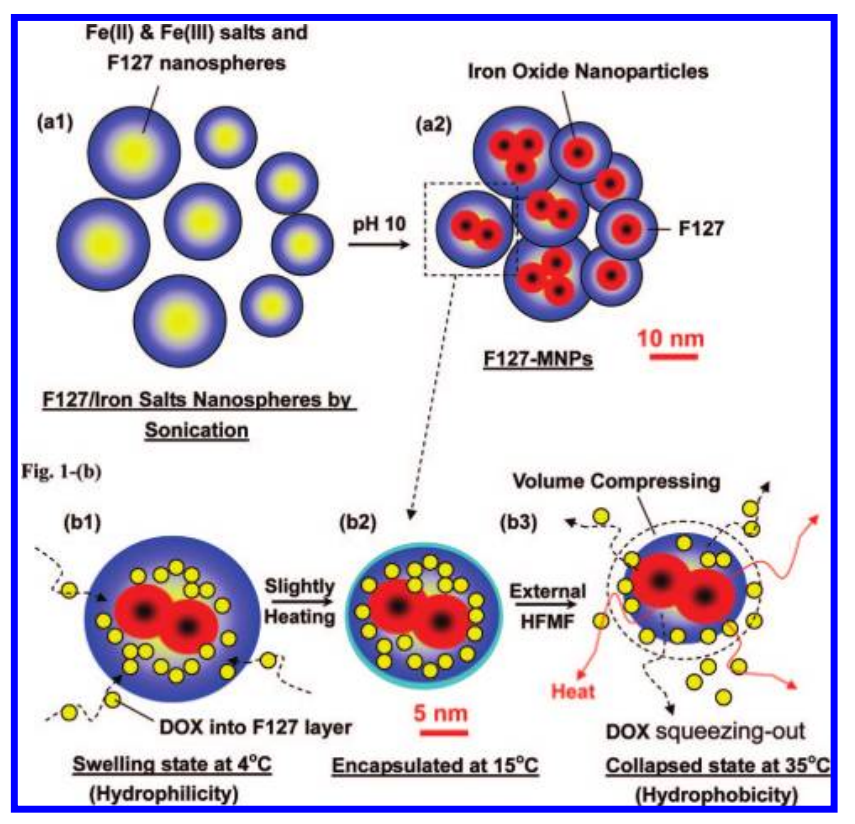

Figure 1. Diagram showing the proposed synthesis process of F127MNPs: (a1) F127/iron salts nanosphere formation by sonication; (a2) F127-MNPs formation after adding ammonia solution to $\mathrm{pH}=10$. Proposed mechanism for drug encapsulation and delivery process of F127-MNPs by HFMF: (b1) drug diffuses into the nanospheres at lower temperature $\left(4^{\circ} \mathrm{C}\right.$, swelling state); (b2) slight volume shrinkage and drug encapsulated in the nanospheres after slight heating to $15^{\circ} \mathrm{C}$; (b3) sharp volume shrinkage with accelerative drug release under HFMF treatment.

made in a similar process with sonication). Later, the $\mathrm{pH}$ was adjusted to 10 by adding ammonia solution $(33 \%)$ to the reactor while stirring. This was followed by heating to $60{ }^{\circ} \mathrm{C}$, where it remained for 30 min. After washing five times with phosphate-buffered saline (PBS, $\mathrm{pH}$ 7.4), subsequent filtering by Acrodisc syringe filters (Aldrich, diameter $25 \mathrm{~mm}$, pore size $0.2 \mu \mathrm{m}$ ), and vacuum-drying, F127MNPs were then successfully prepared, as shown in Figure 1a2.

Doxorubicin (DOX, MW: 580, Sigma, USA), a well-known anticancer drug, was used as a model molecule. DOX was employed because of its amphoteric characteristics; it can dissolve in an aqueous environment (ca. $10 \mathrm{mg} / \mathrm{mL}$ ) or in an organic solvent (high solubility in methanol and chloroform). For incorporation in the F127-MNPs, $2 \mathrm{~mL}$ of an aqueous solution of DOX $(2 \mathrm{mg} / \mathrm{ml})$ was added drop by drop to $3 \mathrm{~mL}$ of an aqueous dispersion of F127-MNPs (100 mg of particles in the $3 \mathrm{~mL}$ PBS solution) while stirring. Stirring was continued overnight at $4{ }^{\circ} \mathrm{C}$ to allow the drug to disperse into the F127-MNPs (see Figure 1b1). Because the F127-MNPs exhibit swelling at lower temperatures $\left(4^{\circ} \mathrm{C}\right)$, DOX easily diffused into the ferrospheres. Then, DOX was well encapsulated under $15^{\circ} \mathrm{C}$ (the collapsing state was at a higher temperature, which formed the protecting-like layer (skin layer) and prevented drug escape), as shown in Figure 1b2. Then, drug-loaded ferrospheres were separated from the unentrapped drug using a magnet. F127-MNPs were washed three times by being resuspended in distilled water and separated, as described above, using the magnetic field. The resultant samples were dialyzed by a dialysis membrane with an MW cutoff at 14000 and stored at $4{ }^{\circ} \mathrm{C}$ before the nanospheres were used.

2.2. Fluorescence and Brunauer-Emmett-Teller (BET) Measurement. Pyrene loading was studied in order to assess the temperature-dependent aggregation within the microgels. A stock solution of $1 \mathrm{mM}$ pyrene in absolute methanol was prepared, from which $2 \mu \mathrm{L}$ was added to $3.0 \mathrm{~mL}$ of a $100 \mathrm{mg}$ F127-shell MNPs aqueous sample. The sample was then allowed to equilibrate for $24 \mathrm{~h}$ at $25^{\circ} \mathrm{C}$. The excitation and emission spectra were recorded

(28) Bromberg, L.; Temchenko, M.; Hatton, T. A. Langmuir 2003, 19, 86758684 .

(29) Bromberg, L.; Barr, D. P. Macromolecules 1999, 32, 3649-3657. 


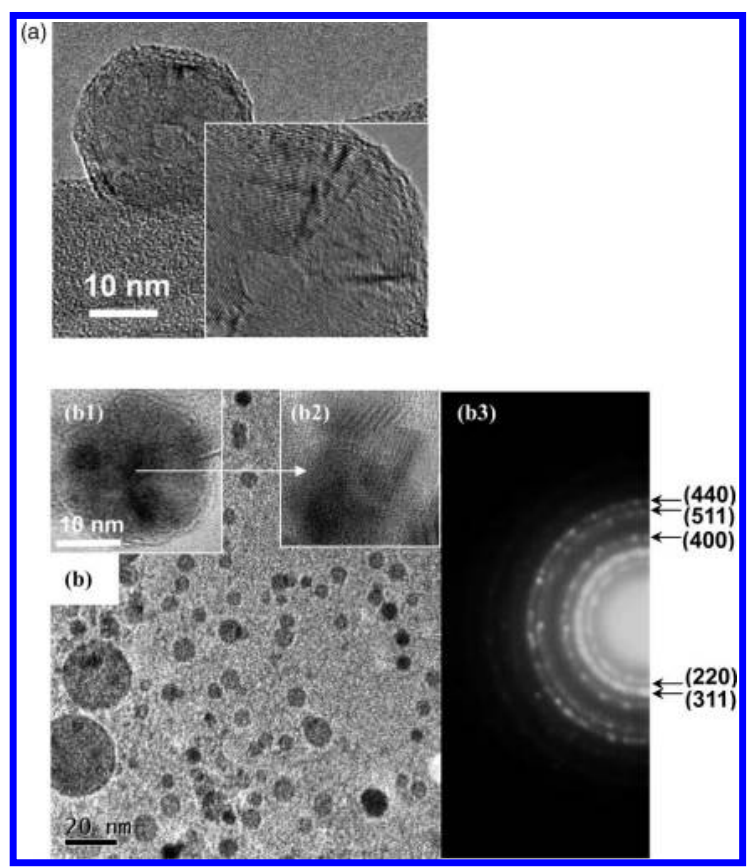

Figure 2. TEM image of the morphology of (a) pure F127 nanospheres and (b) distribution of F127-MNPs. (b1) TEM image of F127-MNPs nanospheres (iron oxide nanoparticles were encapsulated in the F127 nanospheres). (b2) High-resolution TEM image showing the crystalline condition of iron oxide in the F127 nanosphere. (c) Diffraction pattern of F127-MNPs.

using a spectrofluorophotometer (PL) [Kimmon, IK5552R-F, Japan]. A He-Cd laser $(25 \mathrm{~mW})$ was the excitation source. There were five vibrational peaks in the pyrene emission spectra. The ratio of the intensities of the first $(372 \mathrm{~nm})$ to the third $(385 \mathrm{~nm})$ vibrational peak $\left(I_{372} / I_{385}\right)$ in the emission spectra of the monomer pyrene was used to estimate the polarity of the pyrene microenvironment. ${ }^{28,29}$

BET analysis (Quantachrome, NOVA 2000, USA) was conducted using $\mathrm{N}_{2}$ gas absorption isotherms at $77 \mathrm{~K}$, and the pore sizes were calculated following the Barrett-Joyner-Halenda (BJH) method. ${ }^{30}$ The nanospheres $(0.5 \mathrm{~g})$ were dried in vacuum conditions for 1 day before BET analysis. The sample was degassed for $2 \mathrm{~h}$ with a residual vacuum of $0.532 \mathrm{~Pa}$ following BET analysis. ${ }^{13,16}$

2.3. Drug Delivery Test. Drug release behavior of the F127shell MNPs was investigated by applying an HFMF of 50-100 kHz and $15 \mathrm{~kW}$ externally. A cooling water system, copper coil, and HFMF generator were included. The hollow structure of the copper coil was made in order to facilitate cooling water to pass through for temperature control. The copper coil of HFMF was 8 loops, and the strength of the magnetic field was $2.5 \mathrm{k} \mathrm{A} / \mathrm{m} .{ }^{15,16}$ Similar equipment has been reported in the literature. ${ }^{31}$ Because the HFMF generates heat around the copper coil during operation, it was kept at $25^{\circ} \mathrm{C}$ through a cooling water bath to prevent thermally induced interference from the environment. To measure the concentration of drug release, $10 \mathrm{~mL}$ of PBS containing $0.2 \mathrm{~g}$ of F127-MNPs was kept at $15^{\circ} \mathrm{C}$ in the water bath. The absorbance at $482 \mathrm{~nm}^{26}$ was measured by a UV - visible spectrophotometer (8453, Agilent, USA) to determine the concentration of DOX released into the PBS.

\section{Results and Discussion}

3.1. Morphology and Characterization of F127-Shell MNPs. Morphology of F127-shell MNPs was examined by TEM (JEM-2010, JEOL, Japan). Figure 2a shows the TEM images of pure F127 nanospheres, displaying a ring-like structure, which was a phenomenon due to the block-copolymer (Pluronic F127)

(30) Rouquerol, F.; Rouquerol, J.; Sing, K. Adsorption by Powders and Porous Solids; Academic Press: London, 1999.

(31) Derfus, A. M.; von Maltzahn, G.; Harris, T. J.; Duza, T.; Vecchio, K. S.; Ruoslahti, E.; Bhatia, S. N. Adv. Mater. 2007, 19, 3932-3936.

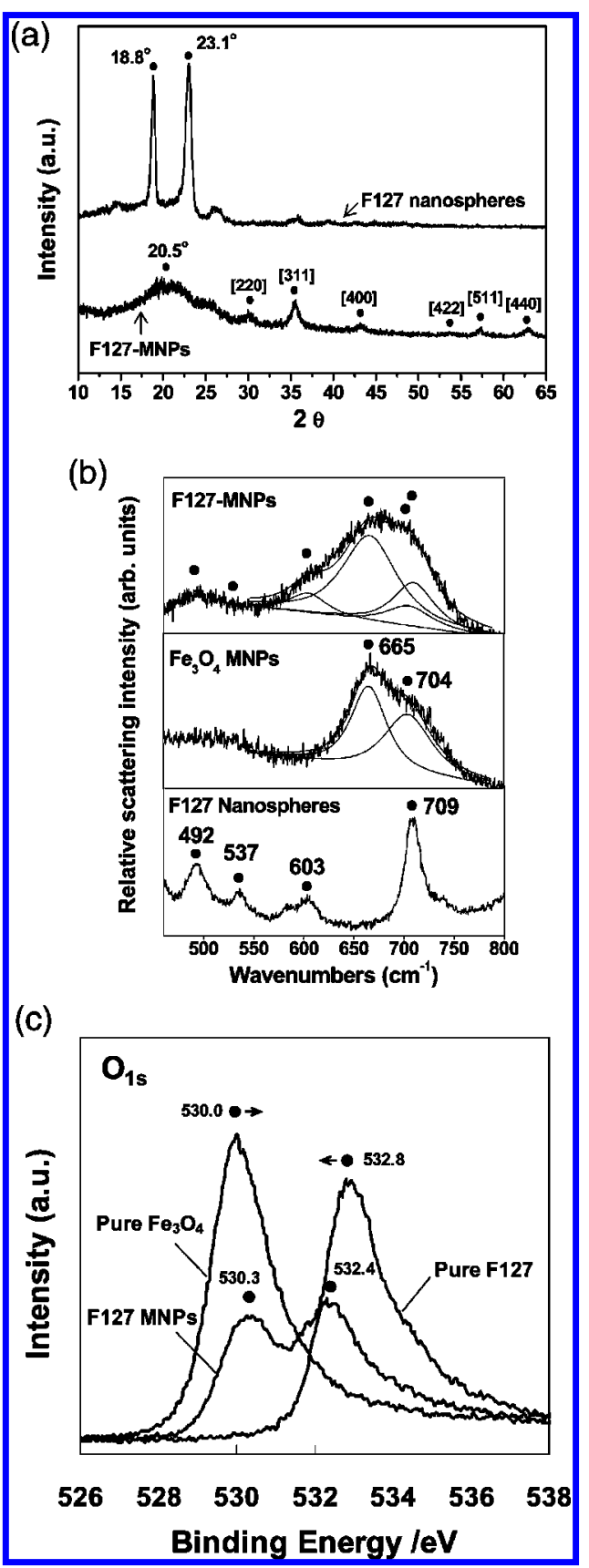

Figure 3. Characterization analysis of F127 and F127-MNP nanospheres: (a) XRD; (b) Raman spectra.

self-assembly. After the precursors of $\mathrm{Fe}$ (II) and Fe(III) salts and the ammonia solution were added, iron oxide nanoparticles started to form by coprecipitation. Figure $2 b$ shows that well-distributed F127-MNPs have a spherical geometry of 10-20 nm diameter. Moreover, under high-resolution TEM [Figure 2b1], the iron oxide nanoparticles were encapsulated in the interior region of F127 nanospheres, and it was a crystalline structure (inset, Figure 2b2). In addition, the selected area diffraction pattern of the F127-shell MNPs showed five planes, namely, [220], [311], [400], [511], and [440], indicating the presence of iron oxide nanoparticles, as well as an amorphous phase, e.g., cloudy ring (see Figure 2b3). This again suggests that the iron oxide nanoparticles were encapsulated by F127 hydrogel.

Figure 3 shows the results of the XRD (M18XHF, Mac Science, Japan) of the F127 nanospheres and F127-MNPs. In Figure 3a, six diffraction peaks at $2 \theta=30.1^{\circ}, 35.6^{\circ}, 43.3^{\circ}, 53.5^{\circ}, 57.2^{\circ}$, and $62.9^{\circ}$ were the characteristic peaks of the crystal plane, which 
was the same as JCPDS $85-1436,{ }^{32}$ suggesting the nanoparticles were $\mathrm{Fe}_{3} \mathrm{O}_{4}$. Furthermore, the XRD spectrum of pure F127 nanospheres showed two characteristic peaks at $2 \theta=18.8^{\circ}$ and $23.1^{\circ}$, representing a high degree of ring-like crystallization of the F127, which was in good agreement with the results of the TEM image [Figure 2a]. In addition, F127-MNPs not only exhibited six characteristic peaks of $\mathrm{Fe}_{3} \mathrm{O}_{4}$ but also showed a broad diffraction pattern, which peaked at $21^{\circ}$. The diffraction pattern was assumed to be due to the presence of iron oxide that deferred the ring-like crystallographic structure of the F127 phase. These results were similar to those of the diffraction pattern of the TEM (cloudy ring), indicating that both phases were intimately contacted.

Raman spectra are potentially more useful than diffraction techniques to track subtle structural differences between the vibration frequencies of $\mathrm{F} 127$ and $\mathrm{Fe}_{3} \mathrm{O}_{4} \mathrm{MNPs}$. Figure $3 \mathrm{~b}$ shows that, although F127 hydrogels were surrounded with iron oxide nanoparticles, a significant difference between these phases can be recognized from the Raman spectra. Both 665 and $704 \mathrm{~cm}^{-1}$ bands, assigned to the characteristic band of $\mathrm{Fe}_{3} \mathrm{O}_{4},{ }^{32}$ were attributed to the vibrational modes. These consisted of the stretching mode of oxygen atoms along $\mathrm{Fe}-\mathrm{O}$ bonds (metal oxide bond), and four characteristic peaks at 492, 537, 603, and $709 \mathrm{~cm}^{-1}$ were assigned to F127 nanospheres. ${ }^{33,34}$ Furthermore, from the sample of F127-MNPs, six peaks, including the peaks from both $\mathrm{F} 127$ and $\mathrm{Fe}_{3} \mathrm{O}_{4} \mathrm{MNPs}$, were observed where the two peaks of 492 and $537 \mathrm{~cm}^{-1}$ were almost combined to form a broad peak. This suggests that there was some interaction between metal oxide and the $-\mathrm{OH}$ group in the $\mathrm{F} 127$ when the iron oxide nanoparticles were formed inside the F127 hydrogel.

In addition, the change in chemical-binding energy in F127MNPs was investigated via XPS (ESCALAB 250, Thermo VG Scientific, West Sussex, U.K.), equipped with $\mathrm{Mg} \mathrm{K} \alpha$ at 1253.6 $\mathrm{eV}$ at the anode. As illustrated in Figure 3c, the binding energy of $\mathrm{O}_{1 \mathrm{~s}}$ was detected from 526.0 to $538.0 \mathrm{eV}$, and the peak of iron oxide was at $530.0 \mathrm{eV} .^{35}$ This peak represented the metal oxide, which was reasonably consistent with peak reported in the literature, i.e., $528-531 \mathrm{eV}$. Also, the $-\mathrm{OH}$ group peak of pure F127 nanospheres was found in the $532.8 \mathrm{eV}$, which was similar to the report of Anderson et al., ${ }^{36}$ showing a highly ordered arrangement of ring-like crystallization of the F127 nanospheres. However, after incorporation into the iron oxide nanoparticles (F127-MNPs), the peak at $532.8 \mathrm{eV}$ shifted to a lower energy, $532.4 \mathrm{eV}$, indicating that the ring-like crystallization of the F127 nanospheres had been deteriorated to an amorphous-like structure as a result of the presence of iron oxide nanoparticles. In the meantime, the peak of the metal oxide in the F127-MNPs shifted to a higher energy, i.e., from 530.0 to $530.3 \mathrm{eV}$, suggesting that some interactions occurred between the metal oxide and the - OH group in the F127 hydrogel when the iron oxide was formed inside the F127 hydrogel. Although the actual mechanism for the shift of the binding energy of metal oxide is unclear at present, it is believed that iron ions can be encapsulated by the F127 hydrogel. This hydrogel promoted a self-assembly of the iron oxide salt to form crystal structure while increasing solution $\mathrm{pH}$

(32) Long, J. W.; Logan, M. S.; Rhodes, C. P.; Carpenter, E. E.; Stroud, R. M.; Rolison, D. R. J. Am. Chem. Soc. 2004, 126, 16879-16889.

(33) Docoslis, A.; Huszarik, K. L.; Papageorgiou, G. Z.; Bikiaris, D.; Stergiou, A.; Georgarakis, E. AAPS J. 2007, 9, E361-E370.

(34) Hendra, P. J.; Agbenyega, J. K. The Raman Spectra of Polymers; John Wiley \& Sons: New York, 1993.

(35) Moulder, J. F.; Stickle, W. F.; Sobol, P. E.; Bomben, K. D. A Reference Book of Standard Spectra for Identification and Interpretation of XPS Data; Physical Electronics, Inc.: Chanhassen, MN, 1995.

(36) Anderson, D.; Nguyen, T.; Lai, P. K.; Amiji, M. J. Appl. Polym. Sci. 2001, 80, 1274-1284.

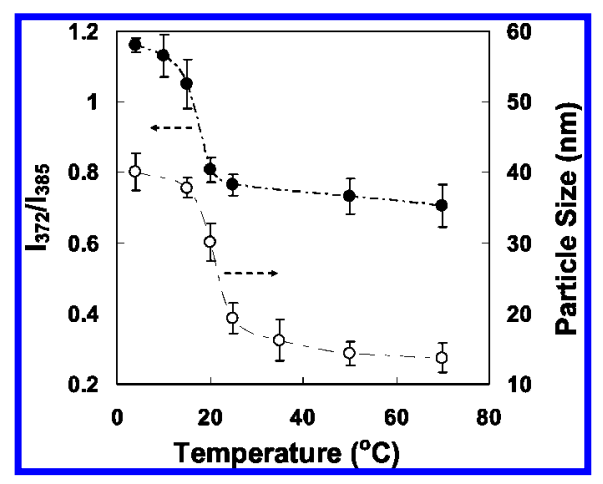

Figure 4. Hydrophilic/Hydrophobic test of F127-MNPs with various temperatures: filled circle curve indicates CMT test by PL (left axis), whereas particle size test by DLS (right axis) is indicated by open circle curve.

upon synthesis, and it thus accompanies some ion-ion interaction to change the binding energy of metal oxide. ${ }^{15}$

3.2. Hydrophilic/Hydrophobic Properties. Pyrene is poorly soluble in a polar environment, but it strongly emits radiation when it is self-aggregated or forms microdomains (i.e., microaggregates) in an aqueous solution. ${ }^{28,29,37}$ The intensity ratio $\left(I_{372} / I_{385}\right)$ of the first peak $(372 \mathrm{~nm})$ and the third peak $(385 \mathrm{~nm})$ of the fluorescence emission spectrum for pyrene has been shown to be sensitive to the polarity of the microenvironment. ${ }^{28,29}$ The reason was that increasing the polarity of the medium induces an increase in the intensity of the first peak, $I_{372}$, corresponding to the forbidden transition, while the intensity of the third peak, $I_{385}$, corresponding to the allowed transition, remains unchanged. ${ }^{38}$ The ratio was higher in a polar medium; for example, in water, $I_{372} / I_{385}=2.0,{ }^{38}$ while, in hexane, $I_{372} / I_{385}=0.6 .^{38}$ Therefore, the CMT, which is the threshold temperature of self-aggregate formation by intra- and/or intermolecular association, could be determined from the change of the $I_{372} / I_{385}$ ratio of pyrene in the presence of polymeric amphiphiles. ${ }^{37}$ Figure 4 shows that the $I_{372} / I_{385}$ ratio in an aqueous suspension of the F127-MNPs varies with temperature between the transition temperatures of 20 and $25{ }^{\circ} \mathrm{C}$, which was the CMT of the F127-MNPs. The $I_{372} / I_{385}$ ratio was higher in water below the CMT, indicating a strongly polar environment. However, above the CMT, the $I_{372} /$ $I_{385}$ ratio dropped to a lower level, as the pyrene partition changed to a more hydrophobic environment as micellar aggregation began to form. These results were comparable with the CMT of pure $\mathrm{F} 127\left(23^{\circ} \mathrm{C}\right)$ in the literature, ${ }^{22,23}$ which meant that the F127-MNPs was more hydrophobic above $25^{\circ} \mathrm{C}$.

Another approach was based on the change of particle size of the F127-MNPs, with respect to temperatures, to indicate the hydrophilicity of the nanoparticles. As shown in Figure 4, the particle size of the F127-MNPs was $40.1 \mathrm{~nm}$ at $4{ }^{\circ} \mathrm{C}$ and 37.8 $\mathrm{nm}$ at $15^{\circ} \mathrm{C}$, but it sharply decreased to $19.3 \mathrm{~nm}$ at $25^{\circ} \mathrm{C}$ and $16.2 \mathrm{~nm}$ at $35^{\circ} \mathrm{C}$, as measured by a DLS instrument (Zetasizer3000 HS, Malvern, U.K.). The particles exhibited a 2- and 2.3fold diameter transition from 15 to $25^{\circ} \mathrm{C}$ and $35^{\circ} \mathrm{C}$, respectively. That was roughly equal to a 10 -fold volume transition from 15 to $35^{\circ} \mathrm{C}$. The 10 -fold volume shrinkage of the F127-MNPs was enough to pump the drug out.

3.3. Drug Delivery Test by HFMF. Figure 5a shows the results of inductive heating of the F127 MNPs, where the temperature was measured by an ethanol thermometer in an

(37) Kalyanasundaram, K.; Thomas, J. K. J. Am. Chem. Soc. 1977, 99, 20392044.

(38) Wang, Y. S.; Liu, L. R.; Jiang, Q.; Zhang, Q. Q. Eur. Polym. J. 2007, $43,43-51$. 


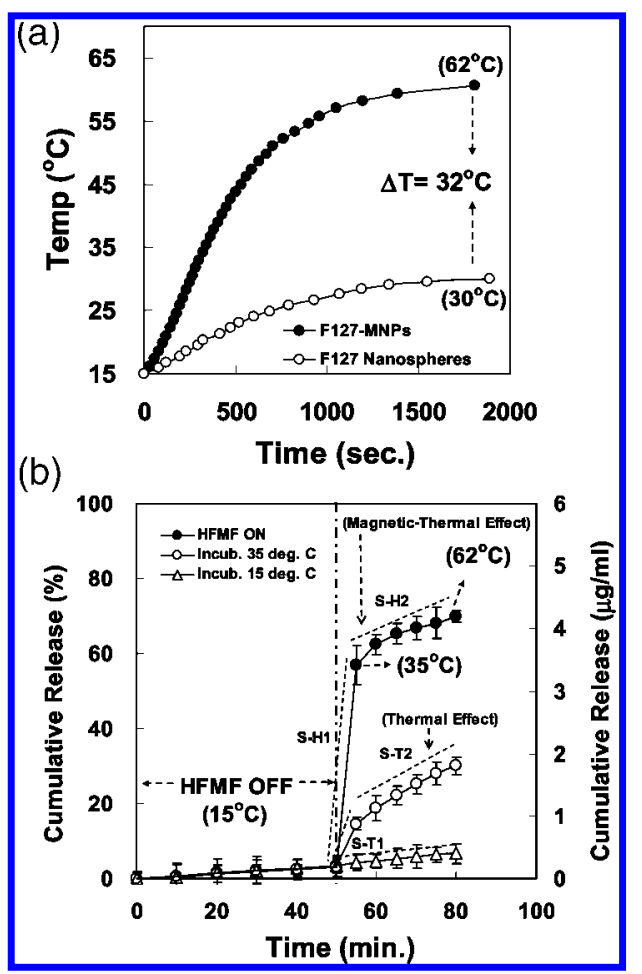

Figure 5. (a) Inductive heating experiments under HFMF treatment; filled circle curve and open circle curve indicate F127-MNPs and F127 nanospheres, respectively; temperature increased to $62^{\circ} \mathrm{C}$ for the $\mathrm{F} 127$ MNPs under HFMF treatment for $60 \mathrm{~min}$, but, for F127 nanospheres, it reached only $30^{\circ} \mathrm{C}$. (b) Drug delivery test of F127-MNPs. Filled circle curve indicates that the samples were first incubated at $15{ }^{\circ} \mathrm{C}$ (water bath) for 50 min and then exposed to HFMF (magnetic-thermal effect). Open circle curve indicates that, after incubation at $15^{\circ} \mathrm{C}$ for $50 \mathrm{~min}$, the samples were incubated at $35^{\circ} \mathrm{C}$ in a water bath (thermal effect, without HFMF), but the open triangle curve means that the samples were incubated at $15^{\circ} \mathrm{C}$ in a water bath $(50+30 \mathrm{~min})$.

aqueous F127-MNPs suspension. Inductive heating is thermal energy induced from the hysteresis loss of ferrites and dependent on the type of remagnetization process in the HFMF. ${ }^{11}$ The results showed that, under the selected field parameters $(50-100 \mathrm{kHz}$ and $15 \mathrm{~kW}$ ), sufficient energy deposition was achieved, and the temperature was substantially increased in the solution within a short period of time. As expected, the temperature increased considerably $\left(62{ }^{\circ} \mathrm{C}\right)$ for the F127-MNPs suspension $(0.2 \mathrm{~g}$ samples in $10 \mathrm{~mL}$ of PBS in the tube) and was approximately 2.0 times higher than that of the pure $\mathrm{F} 127$ nanospheres $\left(30^{\circ} \mathrm{C}\right)$, indicating that this temperature difference $\left(62{ }^{\circ} \mathrm{C}\right.$ to $30^{\circ} \mathrm{C}, \Delta T$ $=32^{\circ} \mathrm{C}$ ) was solely attributed to the inductive heating effect of the iron oxide in the presence of HFMF.

Figure $5 \mathrm{~b}$ shows the relationship between the temperature change and drug release behavior of the F127-MNPs. In the absence of the magnetic field, the drug (DOX) displayed a stable release at a rate of $0.23 \mu \mathrm{g} \mathrm{mL}^{-1} \mathrm{hr}^{-1}$. However, while reaching $35^{\circ} \mathrm{C}$ under magnetic stimulus, the drug release rate increased sharply from 0.23 to $7.94 \mu \mathrm{g} \mathrm{mL}^{-1} \mathrm{hr}^{-1}$ during the first 5-min period (S-T1) and then reduced to $2.23 \mu \mathrm{g} \mathrm{mL}^{-1} \mathrm{hr}^{-1}$ after a further 25-min stimulus (S-T2). The increased release rate when the temperature increased from 15 to $35^{\circ} \mathrm{C}$ (above the CMT) may be attributed to the volume shrinkage of the F127 shell layer. Similar reports have also been addressed in the literature. ${ }^{39}$ For instance, Chung et al. indicated that the PNIPAAm and

(39) Chung, J. E.; Yokoyama, M.; Yamato, M.; Aoyagi, T.; Sakurai, Y.; Okan, T. J. Controlled Release 1999, 62, 115-127.

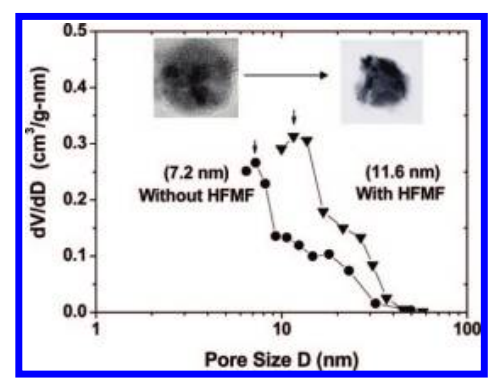

Figure 6. BET analysis on pore size change and TEM images of F127MNPs with and without HFMF. One sample is without HFMF treatment (left side), and the other one is "after" the magnetic filed treatment (right side) in the TEM images.

poly(butyl-methacrylate) polymeric micelles showed reversible structural changes, allowing drug release upon heating/cooling fluctuations with the LCST. These micelles released drug upon heating above the LCST, and the release was accelerated by a volume-shrinkage mechanism, like a pumping effect. The effect was eliminated upon cooling below the LCST.

Furthermore, upon magnetic manipulation, the drug also showed a two-stage release behavior. DOX exhibited a burstlike release for the first $5 \mathrm{~min}$ at a rate of ca. $38.77 \mu \mathrm{g} \mathrm{mL}^{-1} \mathrm{hr}^{-1}$ (S-H1) and then stabilized to ca. $1.84 \mu \mathrm{g} \mathrm{mL}^{-1} \mathrm{hr}^{-1}$ (S-H2) for the following $25 \mathrm{~min}$ of operation. The rate of burst release in the first 5-min period with HFMF was 4.9 times higher than that at $35^{\circ} \mathrm{C}$ in the water bath. The plausible reason was due not only to the volume shrinkage at higher temperature but also to the change in the pore structure of the nanospheres under HFMF. Lu et al. ${ }^{4}$ reported on ferromagnetic cobalt nanoparticles coated with gold shells (Co@Au nanopaticles) embedded into polyelectrolyte capsules. They demonstrated that such magnetic capsules resulted in an increase of wall permeability due to magnetostatic interaction between nanoparticles under oscillating magnetic field. Moreover, a similar mechanism of pore size enlargement under HFMF was also verified by BET analysis in our pervious work. ${ }^{13,16}$

The average pore diameter of the F127-MNPs was determined to be 7.2 and $11.6 \mathrm{~nm}$ without and with $10 \mathrm{~min}$ of exposure to the HFMF, respectively, as shown in Figure 6. The pore size after HFMF treatment was approximately twice that without HFMF treatment, and the pore was enlarged and even broken with HFMF treatment. This indicates that the nanostructure of the F127-MNPs can be manipulated with the HFMF and that the corresponding pore enlargement will induce a rapid drug release, i.e., a burst-like drug release pattern.

The inset TEM image of Figure 6 depicts the collapsed and cracked F127-MNPs. This suggests that the instantaneous drug release under HFMF was due not only to the volume shrinkage but also to the pore rupture of the structure. The collapsed situation was caused by a temperature increase due to generated heat, but the pore rupture might be induced by the violent magnetic vibration of nanoparticles under HFMF. ${ }^{15,16}$ Therefore, HFMFinduced energy can cause the vibration of iron oxide and enlarge pores increasing the permeability of the F127. ${ }^{4}$ Also, the heat energy was conveyed to shrink the F127 hydrogel to pump the drug out. As a result, an instantaneous drug release was achieved using the F127-MNPs by combining the thermal and dynamic energy (vibration of iron oxide nanoparticles) to pump the drug out. Both factors (heat and enlarged pore size) will increase the permeability of the F127, and thus causing the rapid release of the DOX from the F127-MNPs. 


\section{Conclusion}

The dual-functional (magnetic/thermal) drug carriers were successfully fabricated by an in situ process. The F127-MNPs could be used as an effective drug carrier to load an amphoteric drug that could be well partitioned with the nanospheres. In addition, by an externally oscillating magnetic field, a heat source could be produced to induce the instantaneous drug release by a sharp volume transition (10-fold) as a result of a rise in temperature. It could be reasonable to believe that both the heating effect and a rapid release of the anticancer drug upon short exposure to the HFMF offer a dual-functional therapeutic advantage for biomedical application. However, it would be better if the CMT of F127-shell MNPs could be raised to above $37{ }^{\circ} \mathrm{C}$ because it will be more suitable and provide a greater advantage in human body conditions. In other words, the polymer could be designed to operate at a temperature above $37{ }^{\circ} \mathrm{C}$ but to completely cease to act at $37^{\circ} \mathrm{C}$. Therefore, we are trying to modify the Pluronic by covalent bonding with hydrophilic compounds (e.g., PEG or poly(acrylic acid) (PAA)) in order to increase the CMT to $40{ }^{\circ} \mathrm{C}$. In addition, it could also possible to functionalize the F127-shell MNPs with ligands or antibodies to further enhance their potential, including as agents (e.g., quantum dots) for magnetic imaging or tracking.

LA801451V 